\title{
The Application Comparison of Contrast-Enhanced Ultrasound and Contrast-Enhanced Computed Tomography in Radiofrequency Ablation Treatment for Hepatocellular Carcinoma
}

\author{
Zhen Liu, ${ }^{1, *}$ Xiaofei Wang, ${ }^{1, *}$ Lei Xing, ${ }^{2}$ Yan Pan, ${ }^{1}$ and Shengchuan Huang ${ }^{1}$
}

\begin{abstract}
Objective: To compare the application value of contrast-enhanced ultrasound (CEUS) and contrast-enhanced computed tomography (CECT) in radiofrequency ablation (RFA) for treatment of hepatocellular carcinoma (HCC).

Methods: A total of 112 patients with primary HCC were enrolled for the treatment of RFA and they were randomly equally divided into the CEUS group and CECT group. The gender, age, and number and maximum diameter of tumors between the two groups were compared. The median duration of follow-up was 27.0 months and the clinical outcomes were compared.

Results: The average examination time period before ablation, of guiding needle insertion and of ablation in the CEUS group were significantly shorter than those in the CECT group $(p<0.05)$. The complete ablation rate of the CEUS group was significantly higher than that of the CECT group $\left(86.36 \%\right.$ vs. $73.17 \%, \chi^{2}=4.618$, $p=0.032$ ). There was no comparative difference in the incidence of complications (including fever, infection, pain, and liver injury) between the two groups $(p>0.05)$. The relapse rate of the CEUS group was lower and the survival rate was higher than that in the CECT group $(p<0.05)$.

Conclusions: Compared with the CECT, the RFA for treatment of HCC guided by the CEUS shorten the time of examination and treatment. The immediate postoperative assessment can improve the overall complete ablation rate, reduce the relapse rate, and increase the survival rate, which provides insights for the clinical application.
\end{abstract}

Keywords: contrast-enhanced computed tomography, contrast-enhanced ultrasound, hepatocellular carcinoma, radiofrequency ablation

\section{Introduction}

$\mathbf{P}$ atients with hepatocellular carcinoma (HCC), including primary tumor and metastasis, can be treated by transcatheter arterial chemoembolization (TACE), absolute alcohol ablation, radiofrequency ablation (RFA), and microwave ablation with certain application effects. ${ }^{1,2}$ Particularly, ablative treatments currently represent the first-line option for the treatment of early stage unresectable HCC. ${ }^{3}$ The RFA has been widely applied in practice owing to the small trauma and few complications, with high inactivation rate of tumor and low relapse rate. ${ }^{4,5}$ The RFA requires the guidance of ultrasound or computed tomography (CT) imaging to determine the location, number, and size of the tumor, whereas the angle of needle insertion, ablation range, and ablation effect should be also identified. ${ }^{6,7}$ At present, assistant means, including conventional ultrasound, contrast-enhanced ultrasound (CEUS), three-dimensional ultrasound, ${ }^{8,9} \mathrm{CT}$ plain scan, contrast-enhanced computed tomography (CECT), and threedimensional CT have emerged. ${ }^{10,11}$ Notably, the CEUS and

\footnotetext{
${ }^{1}$ Department of Ultrasound, The Affiliated Yantai Yuhuangding Hospital of Qingdao University, Yantai, China.

${ }^{2}$ Department of Ultrasound, Yantai Yuhuangding Hospital, Laishan Branch, Yantai, China.

*Co-first author.
}

Address correspondence to: Shengchuan Huang; Department of Ultrasound, The Affiliated Yantai Yuhuangding Hospital of Qingdao University; No. 20 Yuhuangding East Road, Yantai, Shandong 264000, China

E-mail: shengchuanhuang327@163.com 
the CECT have a higher detection rate of lesions and have good application value in short-term and long-term treatment of HCC. ${ }^{12,13}$ The study thus focused on the comparison of the application value of the CEUS and the CECT in the treatment of HCC.

\section{Objects and Methods}

\section{Object data}

A total of 112 patients with primary HCC in the authors' hospital from January 2013 to June 2015 were enrolled. The study protocol was approved by the Research Ethics Committee of the hospital. The inclusion criteria were: (i) There were no indications of surgical resection and the biopsy was verified as primary HCC; (ii) There was no history of chemoradiotherapy; (iii) $\mathrm{HCC} \leq 3 \mathrm{~cm}$ in diameter and no more than three tumor nodules. (iv) absence of portal/hepatic vein invasion; (v) Child/Pugh Class A or B. (vi) The clinical data were complete and the informed consents were obtained. The exclusion criteria were: (i) The patients received other therapies such as TACE at the same time; (ii) They were complicated with serious liver cirrhosis, viral hepatitis, liver dysfunction, etc.

The patients were randomly divided into CEUS group and CECT group with 56 cases in each group. There were 32 male patients and 24 female patients in the CEUS group, with an average age of $52.6 \pm 13.7$ years, $1.5 \pm 0.6$ tumors on average, and average maximum diameter of $1.5 \pm 1.3 \mathrm{~cm}$. There were 30 male patients and 26 female patients in the CECT group with an average age of $54.5 \pm 16.6$ years, $1.4 \pm 0.5$ tumors on average, and average maximum diameter of $1.6 \pm 1.2 \mathrm{~cm}$. The baseline data of the two groups were compared (Table 1).

\section{Research methods}

The study was completed by the same operation and nursing team. The main processes of the CEUS group were as follows: The E9 color ultrasonic diagnostic apparatus (with transducer frequency at $2.0-5.0 \mathrm{MHz}$ ) of the United States General Electric (GE) Company, real-time CEUS technology with low mechanical index and CPS imaging software, and SonoVue contrast media (59 mg/injection) of

Table 1. Baseline Characteristics of the One Hundred Twelve Patients With Thirty Hepatocellular Carcinomas

\begin{tabular}{lcc}
\hline & \multicolumn{2}{c}{ Value } \\
\cline { 2 - 3 } Characteristics & $\begin{array}{c}\text { CEUS } \\
(\mathrm{n}=56)\end{array}$ & $\begin{array}{c}\text { CECT } \\
(\mathrm{n}=56)\end{array}$ \\
\hline Age (years) mean $\pm \mathrm{SD}$ & $52.6 \pm 13.7$ & $54.5 \pm 16.6$ \\
Sex: male/female & $32 / 24$ & $30 / 26$ \\
Child/Pugh: A/B & $50 / 6$ & $48 / 8$ \\
Size of viable HCC (cm) & $1.5 \pm 1.3$ & $1.6 \pm 1.2$ \\
$\quad$ mean \pm SD & $29(51.8)$ & $30(53.6)$ \\
HCC $\leq 1.5 \mathrm{~cm}(\%)$ & $27(48.2)$ & $26(46.4)$ \\
HCC $>1.5 \mathrm{~cm}(\%)$ &
\end{tabular}

$\mathrm{SD}$, standard deviation; HCC, hepatocellular carcinoma; CEUS, contrast-enhanced ultrasound; CECT, contrast-enhanced computed tomography.
Italian Bracco Corporate were utilized. The liver was examined by conventional ultrasound and the size, location, echo characteristic, and boundary of the lesion were recorded after it was discovered. Around $1.5 \mathrm{~mL}$ of contrast medium was injected through cubital vein at a speed of $1.8 \mathrm{mg} / \mathrm{mL}$ and by means of bolus injection. The changes of echo intensity of the lesions and infusion condition of the contrast medium were observed constantly to ensure that every lesion had complete records for arterial phase, portal phase, and parenchymal phase. Based on the location, range, and blood supply of the lesions displayed by the CEUS, the ablation treatment plan was formulated to determine the mode of needle arrangement and angles of needle insertion. The CEUS was implemented immediately after the RFA to observe whether there were abnormal enhancement areas around or inside the lesions during the portal and arterial phases. Early regression of the portal phase and edge of arterial phase or enhancement inside the tumors indicated that there were residual tumors. The residual lesions could be treated with ablation again.

The main processes of the CECT group were as follows: The 64-slice spiral CT with a scanning thickness of $5 \mathrm{~mm}$ of the United States GE Company was used for volume scanning, and the contrast medium was ULTRAVIST 370 and its injection volume was $50 \mathrm{~mL}$ with an injection speed of $3.0 \mathrm{~mL} / \mathrm{s}$. The CT was selected to display the maximum section of the lesions, and proper layers were dissected. The shortest path with the smallest injury was used as the path for needle insertion. The linear vernier was used to mark the puncture points. The channel for needle insertion was simulated. The angles and directions of needle insertion were designed. The optimum depth of needle insertion was measured. The gentian violet was used to mark the puncture points. After the local anesthesia was successful, the suction biopsy needle 18-21 G was used to insert the needle step by step under the CT guidance according to the simulated angles of needle insertion until the radiofrequency needle reached the target. The patients were requested to hold their breath during the puncture and then they were scanned again with CT to verify that the puncture was satisfied. After the puncture was in place, the inner trocar was pushed out and the cluster electrode needles were unfolded. The patients were scanned again with CT to verify that the unfolding of electrodes was satisfied. The CECT was implemented 1 month after the operation to judge the ablation effect. The low density, which was shown by the three phases of the ablation lesion were determined as complete ablation. A part of the arterial phase of the ablation lesion showed a high enhancement, whereas the regression of the portal and parenchymal phases showed a low-density lesion, and it was determined as an incomplete ablation.

The main processes of the RFA were as follows: The RF2000 RFA instrument with electrode frequency $500 \mathrm{kHz}$ and power $100 \mathrm{~W}$. The United States Resolution Trust Corporation and the hollow cooling radiofrequency needle with cold circulating system was used. After the local anesthesia and puncture were successful, the cold circulating radiofrequency needles were placed in the area where the distance from the interior lesion to the edge of the lesion was $<1.0 \mathrm{~cm}$; the needle stitch of the radiofrequency needles was $<2.0 \mathrm{~cm}$. After the needle point reached the bottom of the tumor and the basal anesthesia took effect, the 
instrument was turned on for treatment and then it could be seen that the lesions were completely covered by strong echoes. For the lesions near the liver capsule, such as diaphragm or intestinal canal, the method of directly injecting normal saline under the liver capsule could be adopted to separate the diaphragm or nearby organs. when the radiofrequency instrument indicated that the timely impedance and the absorbed energy value of the ablation lesion had met the requirements, the needle was withdrawn, the instrument was turned off, and the treatment was finished. The lesions with a diameter $<3.0 \mathrm{~cm}$ could be ablated at once, whereas those with a diameter more than $3.0 \mathrm{~cm}$ were needed to be ablated repeatedly from multiple points and directions. The ablation range covered $5-10 \mathrm{~mm}$ of the tumor edge.

\section{Observation indexes}

The time of RFA, complete ablation rates, and incidences of complications of the two groups were compared and the relapse rates and survival rates were followed. The duration of follow-up was 12.0-40.0 months and the median duration was 27.0 months.

\section{Statistical methods}

SPSS20.0 software was used for statistical analysis and the data were presented as mean \pm standard deviation. Independent-samples $t$ test was used for comparison between groups. The enumeration data were presented as cases or $(\%)$ and the $\chi^{2}$ test was used for comparison between groups. Kaplan-Meier survival analysis and the log-rank test were performed between two groups. $p<0.05$ was considered to be statistically significant.

\section{Results}

\section{Comparisons of RFA time between the two groups}

The baseline characteristics of 112 patients in CEUS and CECT groups are summarized in Table 1. There were no significant differences of age, sex, Child/Pugh, tumor size, and range between two groups. The average examination time period before ablation, of guiding needle insertion and of ablation treatment of the CEUS group were significantly shorter than those of the CECT $(p<0.05)$ (Table 2).

\section{Comparisons of complete ablation rates}

According to the complete ablation determined by the CECT examination 1 month after the operation, 76 of 88 $(86.36 \%)$ lesions in the CEUS group $(n=46)$ were com-

TABle 2. Comparisons of RAdiofrequency Ablation Time Between the Two Groups (Minutes)

\begin{tabular}{lccc}
\hline & $\begin{array}{c}\text { Check time } \\
\text { before } \\
\text { ablation }\end{array}$ & $\begin{array}{c}\text { Time of } \\
\text { guiding } \\
\text { needle } \\
\text { insertion }\end{array}$ & $\begin{array}{c}\text { Time of } \\
\text { ablation } \\
\text { treatment }\end{array}$ \\
\hline CEUS group & $15.6 \pm 5.4$ & $3.4 \pm 0.8$ & $2.7 \pm 0.6$ \\
CECT group & $24.7 \pm 8.2$ & $6.5 \pm 1.4$ & $3.3 \pm 0.9$ \\
$T$ & 5.063 & 4.528 & 4.126 \\
$P$ & 0.009 & 0.016 & 0.023 \\
\hline
\end{tabular}

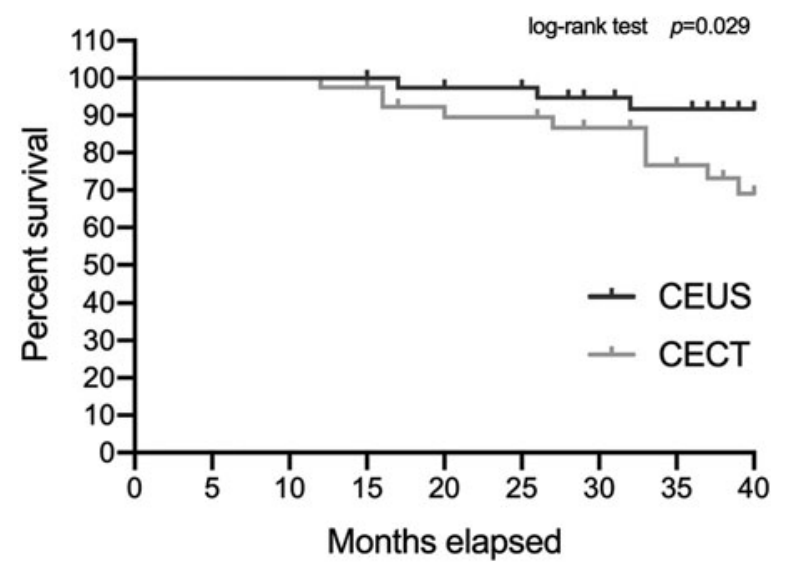

FIG. 1. Kaplan-Meier survival curve for patients with hepatocellular carcinoma treated with radiofrequency ablation from CEUS and CECT groups. CEUS, contrastenhanced ultrasound; CECT, contrast-enhanced computed tomography.

pletely ablated and 60 of $82(73.17 \%)$ lesions in the CECT group $(n=41)$ were completely ablated. The complete ablation rate of the CEUS group was significantly higher than that of the CECT group $\left(\chi^{2}=4.618, p=0.032\right)$.

\section{Comparisons of incidences of complications}

There were two cases of fever (body temperature higher than $38.5^{\circ} \mathrm{C}$ ), one case of infection, three cases of pain (visual analog scale $\geq 2$ points), and two cases of liver injury (alanine aminotransferase increased by at least 1.5 times) in the CEUS group and the incidence of postoperative side effects was $14.29 \%(8 / 56)$. However, the number of cases of fever, infection, pain, and liver injury was 3,2, 2, and 3, respectively, in the CECT group and the relative incidence was $17.86 \%(10 / 56)$. There was no significant difference between the two groups $\left(\chi^{2}=0.265, p=0.607\right)$. The complications were controlled after symptomatic treatments.

\section{Comparisons of followed-up relapse rates and survival rates}

We further analyzed the followed-up relapse rates and survival rates in patients with complete ablation from two groups. Survival probability was estimated by KaplanMeier method, and log-rank test showed that there was significant difference between two groups $(p<0.05)$ (Fig. 1). Of note, the relapse rate of the CEUS group was statistically

Table 3. Comparisons of Followed-Up Relapse Rates and Survival Rates [Case (\%)]

\begin{tabular}{lcccc}
\hline Group & $\begin{array}{c}\text { Total } \\
\text { cases }\end{array}$ & $\begin{array}{c}\text { Complete } \\
\text { ablation } \\
\text { cases }\end{array}$ & $\begin{array}{c}\text { Relapse } \\
\text { rate }\end{array}$ & $\begin{array}{c}\text { Survival } \\
\text { rate }\end{array}$ \\
\hline CEUS group & 56 & 46 & $4(8.70)$ & $43(93.48)$ \\
CECT group & 56 & 41 & $12(29.27)$ & $32(78.05)$ \\
$\chi^{2}$ & & 1.287 & 6.113 & 4.340 \\
$P$ & & 0.257 & 0.013 & 0.037 \\
\hline
\end{tabular}


lower than that of the CECT group, and the survival rate was significantly increased $(p<0.05)$ (Table 3$)$.

\section{Discussion}

The HCC diagnosed by the CEUS is presented as spherical circumvolution, diffuse or mild enhancement in early artery phase, rapid clearance of contrast media in portal phase, and typical fast in and fast out. Compared with conventional ultrasound, the CEUS increased the detection rate of HCC, which is equivalent with the CECT. ${ }^{14}$ In particular, CEUS has more advantages in diagnosing lesions of $\mathrm{HCC}$ with diameter $<1 \mathrm{~cm}$ and the diagnostic rate was increased from $53 \%$ to $94 \% .{ }^{15}$ The CEUS can clearly determine the number, nature, range, feeding vessel, size, infiltration range, and other information of the lesions of HCC and provide fairly detailed imaging basis for determination of treatment plan to precisely ensure if the lesions are removed thoroughly.

Our data showed that the average examination time period before ablation, of guiding needle insertion, and of ablation treatment of the CEUS group were significantly shorter than those of the CECT group. The CEUS presented a higher sensitivity to the echoes of normal hepatic tissues, HCC, and tumor boundary than CECT. The paths of needle insertion and dissection and depth of needle insertion could be precisely provided by analyzing the hemoperfusion condition of tumor in various phases through the contrast media. During and after the operation, the CEUS can be used as real-time guidance and examination, and it can accurately assess the needle insertion, unfolding electrodes and ablation effects. Therefore, it serves as a convenient and flexible way to guide ablation at multiple points and locations. ${ }^{16}$ Meanwhile, CEUS contributes to a comparatively safe strategy due to the fast imaging, consumes small volume of contrast media, and causes slight injury to patient's liver and kidney. ${ }^{17}$ The shortened treatment time posed an important significance for improving patient's tolerance degree, reducing complications, extending ablation time, and improving ablation effects. ${ }^{18}$ The complete ablation rate of the CEUS group was significantly higher than that of the CECT group, which might benefit from immediate postoperative examination, assessment of ablation effects, as well as the rapid and repeated ablations. ${ }^{19}$ There was a congestion and edema region appearing around the tissues undergoing RFA and it is hard to distinguish the congestion zone from tumor residuals through the CECT. The RFA effects could only be assessed 1 month after the congestion zone had disappeared. However, the CEUS could accurately recognize the residual tumors with incomplete ablation as early as possible so that it is possible to timely inactivate the lesions of HCC in one operation. ${ }^{20}$ As for lesions with a diameter exceeding $5.0 \mathrm{~cm}$, it is quite challenging to realize complete ablation through one needle insertion, thus the ablations are mostly realized by alternate or repeated needle insertions. The CEUS could implement supplementary RFA through the timely detection of the tumor residuals. ${ }^{21}$ The accuracy rate of the CEUS judging the complete necrosis of the tumors after ablation reaches about $95.0 \% .^{22}$ No comparative difference in the incidence of complication between the two groups suggests favorable security. Our result indicated that the relapse rate of the CEUS group was lower than that of the CECT group, the survival rate was increased, and the judgment of early treatment effects after ablation, which is the key factor of reducing the relapse rate. $^{23}$

The limitation of CEUS still exists. For example, the duration of the arterial phase is short, which is not beneficial to comprehensively and objectively assess the blood supply of tumors. Furthermore, the observation of the lesion section requires improvement and the three-dimensional CEUS can enable the three-dimensional and multiangle observation. It is still very difficult to analyze several lesions in the same phase, and the detection of lesions in deep locations (distance to the body surface exceeds $12 \mathrm{~cm}$ ) remains to be further optimized. In addition, RFA was generally performed under the guidance of ultrasound instead of CEUS. Although CT is also applied in some cases to guide RFA, ultrasound-guided RFA is definitely the mainstay of treatment. These data provide a future basis for fusion imaging guidance system using ultrasound and CT.

\section{Conclusions}

To sum up, CEUS can shorten the duration of examination and treatment in terms of guiding RFA of HCC compared with the CECT. The immediate postoperative assessment can improve the overall complete ablation rate, reduce the relapse rate, and increase the survival rate, which provides a valuable method for the clinical application.

\section{Disclosure Statement}

There are no existing financial conflicts.

\section{Funding Information}

This work was supported by ultrasound elastic shear wave imaging technology combined with magnetic resonance dynamic enhanced scanning to evaluate the efficacy of radiofrequency ablation of primary hepatocellular carcinoma (2016WS027).

\section{References}

1. Qiu GP, Liu J. MicroRNA gene polymorphisms in evaluating therapeutic efficacy after transcatheter arterial chemoembolization for primary hepatocellular carcinoma. Genet Test Mol Biomarkers 2016;20:579.

2. Nouso K, Kariyama K, Nakamura S, et al. Application of radiofrequency ablation for the treatment of intermediatestage hepatocellular carcinoma. J Gastroenterol Hepatol 2017;32:695.

3. Facciorusso A, Serviddio G, Muscatiello N. Local ablative treatments for hepatocellular carcinoma: An updated review. World J Gastrointest Pharmacol Ther 2016;7:477.

4. Lee SK, Song MJ. Drop metastasis from hepatocellular carcinoma after percutaneous radiofrequency ablation therapy. Korean J Intern Med 2017;32:943.

5. Sucandy I, Cheek S, Golas BJ, et al. Longterm survival outcomes of patients undergoing treatment with radiofrequency ablation for hepatocellular carcinoma and metastatic colorectal cancer liver tumors. HPB (Oxford) 2016; 18:756.

6. $\mathrm{Wu} \mathrm{J}$, Lin S, Wu W, et al. Clinical application of ultrasound-guided radiofrequency ablation for primary he- 
patocellular carcinoma near the liver surface. Zhonghua Zhong Liu Za Zhi 2015;37:933.

7. Tanaka T, Ikeda K, Sorin Y, et al. Three-dimensional imaging using contrast-enhanced and three-dimensional ultrasound techniques in the ablative zone treated with a multipolar radiofrequency ablation system for hepatocellular carcinoma. Oncology 2016;90:255.

8. Leen E, Kumar S, Khan SA, et al. Contrast-enhanced 3D ultrasound in the radiofrequency ablation of liver tumors. World J Gastroenterol 2009;15:289.

9. Rajesh S, Mukund A, Arora A, et al. Contrast-enhanced US-guided radiofrequency ablation of hepatocellular carcinoma. J Vasc Interv Radiol 2013;24:1235.

10. Pan T, Xie QK, Lv N, et al. Percutaneous CT-guided radiofrequency ablation for lymph node oligometastases from hepatocellular carcinoma: A propensity scorematching analysis. Radiology 2017;282:259.

11. Inoue T, Kudo M, Hatanaka K, et al. Usefulness of contrastenhanced ultrasonography to evaluate the post-treatment responses of radiofrequency ablation for hepatocellular carcinoma: Comparison with dynamic CT. Oncology 2013; 84(suppl1):51.

12. Ricci P, Cantisani V, Drudi F, et al. Is contrast-enhanced US alternative to spiral CT in the assessment of treatment outcome of radiofrequency ablation in hepatocellular carcinoma? Ultraschall Med 2009;30:252.

13. Zheng SG, Xu HX, Lu MD, et al. Role of contrast-enhanced ultrasound in follow-up assessment after ablation for hepatocellular carcinoma. World J Gastroenterol 2013;19:855.

14. Du J, Li HL, Zhai B, et al. Radiofrequency ablation for hepatocellular carcinoma: Utility of conventional ultrasound and contrast-enhanced ultrasound in guiding and assessing early therapeutic response and short-term follow-up results. Ultrasound Med Biol 2015;41:2400.

15. Chan AK, Hegarty C, Klass D, et al. The role of contrastenhanced ultrasound in guiding radiofrequency ablation of hepatocellular carcinoma: A retrospective study. Can Assoc Radiol J 2015;66:171.

16. Lekht I, Gulati M, Nayyar M, et al. Role of contrastenhanced ultrasound (CEUS) in evaluation of thermal ablation zone. Abdom Radiol (NY) 2016;41:1511.

17. Minami Y, Kudo M. Imaging modalities for assessment of treatment response to nonsurgical hepatocellular carcinoma therapy: Contrast-enhanced US, CT, and MRI. Liver Cancer 2015;4:106.

18. Gao Y, Zheng DY, Cui Z, et al. Predictive value of quantitative contrast-enhanced ultrasound in hepatocellular carcinoma recurrence after ablation. World J Gastroenterol 2015;21:10418.

19. Bartolotta TV, Taibbi A, Matranga D, et al. 3D versus 2D contrast-enhanced sonography in the evaluation of therapeutic response of hepatocellular carcinoma after locoregional therapies: Preliminary findings. Radiol Med 2015; 120:695.

20. Palmieri VO, Santovito D, Marano G, et al. Contrastenhanced ultrasound in the diagnosis of hepatocellular carcinoma. Radiol Med 2015;120:627.

21. Xu X, Luo L, Chen J, et al. Acoustic radiation force impulse elastography for efficacy evaluation after hepatocellular carcinoma radiofrequency ablation: A comparative study with contrast-enhanced ultrasound. Biomed Res Int 2014;2014:901642.

22. Catalano O, Izzo F, Vallone P, et al. Integrating contrastenhanced sonography in the follow-up algorithm of hepatocellular carcinoma treated with radiofrequency ablation: Single cancer center experience. Acta Radiol 2015;56: 133.

23. Wiggermann $\mathrm{P}$, Brünn $\mathrm{K}$, Rennert $\mathrm{J}$, et al. Monitoring during hepatic radiofrequency ablation (RFA): Comparison of real-time ultrasound elastography (RTE) and contrastenhanced ultrasound (CEUS): First clinical results of 25 patients. Ultraschall Med 2013;34:590. 